THE ASTROPHYSICAL JouRNAL, 337:399-407, 1989 February 1

(C) 1989. The American Astronomical Society. All rights reserved. Printed in U.S.A.

\title{
NON-EQUILIBRIUM IONIZATION EFFECTS IN THE SUPERNOVA REMNANT RCW 86
}

\author{
J. J. Claas, ${ }^{1}$ A. Smith, ${ }^{2}$ J. S. KaAstra, ${ }^{1}$ P. A. J. De Korte, ${ }^{3}$ And A. Peacock ${ }^{2}$ \\ Received 1987 September 29; accepted 1988 July 11
}

\begin{abstract}
X-ray images show that the SNR RCW 86 consists of two emission regions. The electron temperature of both two regions is $3.4 \mathrm{keV}$. The low-energy flux $(0.2-2 \mathrm{keV})$ is strongly enhanced compared to a simple thermal bremsstrahlung spectrum with $T_{e}=3.4 \mathrm{keV}$ fitted to the $2-10 \mathrm{keV}$ data. This enhancement is most likely caused by non-equilibrium ionization effects in which case our data of RCW 86 are consistent with a Type I remnant for which the northeast part is in the Sedov phase. The large brightness of the southwest part is most probably caused by a recent collision of the shock with higher density clouds.
\end{abstract}

Subject headings: nebulae: individual (RCW 86) - nebulae: supernova remnants - X-rays: sources

\section{INTRODUCTION}

RCW 86 is most likely the remnant of the supernova recorded by Chinese astronomers in A.D. 185 (Clark and Stephenson 1977). It has been rediscovered as the radio source MSH 14-63 (also G315.4-2.3) (Hill 1967). High-resolution maps at 408 and $5000 \mathrm{MHz}$ by Caswell, Clark, and Crawford (1975) clearly show a nearly circular shell with an angular diameter of $\sim 40^{\prime}$.

Optically, the remnant shows evidence of a number of filaments (Ruiz 1981), which coincide with the radio emission.

The first X-ray observation was reported by Naranan et al. (1977) using an experiment sensitive in the energy range 0.5-2.5 $\mathrm{keV}$ on the Apollo-Soyuz mission. The observed spectrum was interpreted by a thermal bremsstrahlung spectrum with a temperature of $\sim 0.4 \mathrm{keV}$. Winkler (1978), using the M.I.T. experiment on $\operatorname{OSO} 7$, sensitive in the energy range $1-60 \mathrm{keV}$, needs two thermal bremsstrahlung components with temperatures $T_{1} \approx 0.22 \mathrm{keV}$ and $T_{2}>5 \mathrm{keV}$, in order to explain the spectrum observed.

Spatially resolved X-ray images of RCW 86 (Pisarski, Helfand, and Kahn 1984) obtained by the Einstein imaging proportional counter show a strong correlation with the radio maps. Nugent et al. (1984), using the HEAO $1 \mathrm{~A} 2$ experiment, detect a strong $\mathrm{FeK} \alpha$ emission line at $\sim 6.7 \mathrm{keV}$, thereby proving the thermal origin of the X-ray emission.

The distance, as discussed by several authors, is between 0.7 and $3.2 \mathrm{kpc}$ (Milne 1970; Ilovaisky and Lequeux 1972; Clark and Caswell 1976; Poveda and Woltjer 1968; Westerlund 1969; Ruiz 1981; Minkowski 1968).

This paper describes the analysis and interpretation of data obtained with the European X-ray satellite EXOSAT. For the interpretation of these data we use both a collisional ionization equilibrium (CIE) plasma model and a non-equilibrium ionization (NEI) plasma model. The CIE model is appropriate for a low-density, optically thin plasma and includes $\mathrm{ff}, \mathrm{bf}, \mathrm{bb}$, line, and two-photon emission (Mewe, Gronenschild, and Van den Oord 1985). Contrary to the CIE model, the NEI model accounts for the finite time required for reaching ionization equilibrium (Itoh 1977, 1978, 1979; Hamilton, Sarazin, and Chevalier 1983; Jansen 1988).

\footnotetext{
${ }^{1}$ Laboratory for Space Research Leiden, Leiden, the Netherlands.

2 Space Science Department, ESTEC, Noordwijk, the Netherlands.

${ }^{3}$ Laboratory for Space Research Utrecht, Utrecht, the Netherlands.
}

\section{INSTRUMENTS AND OBSERVATIONS}

The present observations of RCW 86 have been made by two experiments on board EXOSAT. These experiments have been thoroughly described elsewhere (de Korte et al. 1981; Turner, Smith, and Zimmerman 1981). Here we only mention some characteristic features of these experiments.

The two imaging telescopes LE1 and LE2, sensitive in the low-energy (LE) range $0.05-2 \mathrm{keV}$, have a field of view two degrees in diameter and an on-axis spatial resolution of $\sim 18^{\prime \prime}$, decreasing toward the edge of the field of view. These telescopes can be used with several filters, e.g., a $3000 \AA$ Lexan (3Lx), an Aluminum-Parylene (AlPa) and a Boron (Bo) filter. Differences in the spectral response of these filters provide the possibility to carry out some coarse spectrophotometry with this experiment.

The medium-energy (ME), large area, proportional counter array provides spectral information for the energy range 1.5-20 $\mathrm{keV}$. It is composed of eight counters grouped into four separate so-called quadrants and has a field of view $45^{\prime}$ in diameter (FWHM) and an effective area of $\approx 1500 \mathrm{~cm}^{2}$. If, as in the case of RCW 86, the size of the remnant is similar to the ME FWHM, some rough spatial information can be obtained by observing at different pointing directions.

Observations with different pointing directions were carried out on RCW 86. The observations used for the present analysis are summarized in Table 1.

\section{DATA ANALYSIS}

\section{a) Spatial Information}

The LE images for the three different filters have been obtained by addition of the data collected from different pointings. Before addition each image has been corrected for background and variations in the effective area across the detector. The background spatial distribution is taken from deep field images of high statistical quality, while its intensity is normalized to regions outside RCW 86 . The three images are rebinned to $64^{\prime \prime}$ per pixel in order to obtain a uniform spatial resolution over the whole remnant, as well as to improve the statistical quality.

The error calculated for each pixel includes statistical and systematic effects. The systematic error has been determined after a detailed analysis of the background subtraction accuracy and equals $\approx 1 \%(1 \sigma)$ of the raw background level. 
TABLE 1

SUMMARY OF LE AND ME OBSERVATIONS

\begin{tabular}{|c|c|c|c|c|c|c|c|}
\hline $\begin{array}{l}\text { Pointing } \\
\text { Number }\end{array}$ & $\begin{array}{l}\text { Date of } \\
\text { (yr/day) }\end{array}$ & $\begin{array}{c}\text { Observing } \\
\times 10^{3} \mathrm{~s}\end{array}$ & $l_{I I}$ & $b_{I I}$ & LE1 & LE2 & ME \\
\hline $1 \ldots \ldots \ldots \ldots \ldots \ldots$ & $1984 / 107$ & 7 & 315.46 & -1.86 & $3 \mathrm{Lx}$ & $\ldots$ & slew \\
\hline $2 \ldots \ldots \ldots \ldots \ldots \ldots$ & $1984 / 104$ & 26 & 315.27 & -2.00 & $3 \mathrm{Lx}$ & & slew \\
\hline $3 \ldots$ & $1983 / 239$ & 11 & 315.67 & -2.11 & & $3 \mathrm{Lx}$ & slew \\
\hline$\ldots \ldots \ldots$ & $1985 / 089$ & 9 & 315.43 & -2.18 & $\mathrm{AlPa}$ & $\ldots$ & swop \\
\hline$\ldots \ldots \ldots$ & $1985 / 089$ & 8 & 315.28 & -2.20 & $\mathrm{AlPa}$ & $\ldots$ & swop \\
\hline $6 \ldots \ldots \ldots \ldots \ldots \ldots \ldots$ & $1985 / 089$ & 5 & 315.61 & -2.23 & $\mathrm{AlPa}$ & & swop \\
\hline $7 \ldots \ldots \ldots \ldots \ldots \ldots$ & $1983 / 240$ & 12 & 315.65 & -2.31 & .. & $3 \mathrm{Lx}$ & slew \\
\hline $8 \ldots \ldots$ & $1983 / 257$ & 11 & 315.23 & -2.33 & & $\mathrm{AlPa}$ & slew \\
\hline $9 \ldots$ & $1984 / 060$ & 18 & 315.19 & -2.40 & Bo & $\ldots$ & slew \\
\hline $10 \ldots \ldots \ldots \ldots \ldots \ldots$ & $1985 / 089$ & 9 & 315.60 & -2.40 & $\mathrm{AlPa}$ & $\ldots$ & slew \\
\hline $11 \ldots \ldots \ldots \ldots \ldots \ldots$ & $1984 / 059$ & 10 & 315.23 & -2.43 & Bo & $\ldots$ & slew \\
\hline $12 \ldots \ldots \ldots \ldots \ldots \ldots$ & 1985/089 & 6 & 315.36 & -2.55 & $\mathrm{AlPa}$ & $\ldots$ & swop \\
\hline $13 \ldots \ldots \ldots \ldots \ldots \ldots$ & $1984 / 051$ & 3 & 315.36 & -2.24 & $3 \mathrm{Lx}$ & $\ldots$ & no $\mathrm{ME}$ \\
\hline $14 \ldots \ldots \ldots \ldots \ldots \ldots$ & $1984 / 051$ & 8 & 315.36 & -2.24 & Bo & $\ldots$ & no $\mathrm{ME}$ \\
\hline $15 \ldots \ldots \ldots \ldots \ldots \ldots$ & $1984 / 053$ & 8 & 315.44 & -2.46 & Bo & $\ldots$ & no $\mathrm{ME}$ \\
\hline
\end{tabular}

Figure $1 a$ shows the $\mathrm{X}$-ray image for the $3 \mathrm{Lx}$ filter. This image reveals the following structure:

1. The remnant has an incomplete shell-like structure, with essentially no emission from the central part, placing it, as far as the LE image is concerned, among the shell-like type of remants.

2. The brightness of the southwest part of the remnant is appreciably larger than that of the northeast part by about a factor of 4 .

3. The northeast part can be fitted by a circle with a radius of $21^{\prime} \pm 1^{\prime}$ centered at position $\alpha=14^{\mathrm{h}} 38^{\mathrm{m}} 55^{\mathrm{s}}$ and $\delta=-62^{\circ} 17^{\prime} 40^{\prime \prime}$ (see $\S$ IVb).

4. The extremum of the southwest part falls on the circle which fits the northeast.

5. However, the radius of curvature of the southwest part is appreciably smaller than the northeast part.

6. Also, the annular width of the southwest part is too wide to be caused by limb brightening only.

In Figure $1 a$ the definitions of the northeast and southwest part of the remnant are indicated. The northeast part is defined as the region with a position angle between $-50^{\circ}$ and $+130^{\circ}$ with a radius smaller than $21^{\prime}$. The southwest part is defined as the region with a position angle between $+187^{\circ}$ and $+310^{\circ}$ and radii extending to the lowest closed contour level. The exact definitions are mainly based on statistical grounds (see $\S \mathrm{IV} b)$.

In Figure $1 b$, which shows the $3 \mathrm{Lx}$ image after being smoothed to a resolution of $3^{\prime}$ (FWHM), the structure of the northeast part is seen more clearly.

Table 2 gives the count rates determined from the three images. In order to search for spectral morphology, the countrate ratios of $\mathrm{AlPa} / 3 \mathrm{Lx}$ and $\mathrm{Bo} / 3 \mathrm{Lx}$ were also calculated as a function of radial distance and position angle by dividing the

TABLE 2

SUMMARY OF LE COUNT RATES

\begin{tabular}{ccccc}
\hline \hline $\begin{array}{c}\text { Filter } \\
\text { (ratio) }\end{array}$ & $\begin{array}{c}\text { NE Part } \\
\text { (counts s }^{-1} \text { ) }\end{array}$ & $\begin{array}{c}\text { SW Part } \\
\text { (counts s }^{-1} \text { ) }\end{array}$ & $\begin{array}{c}\text { Total } \\
\text { (counts s }^{-1} \text { ) }\end{array}$ & $\begin{array}{c}\text { NE Part } \\
\text { SW Part }\end{array}$ \\
\hline $3 \mathrm{Lx} \ldots \ldots \ldots \ldots$ & $0.091 \pm 0.008$ & $0.348 \pm 0.008$ & $0.439 \pm 0.011$ & $0.26 \pm 0.02$ \\
$\mathrm{AlPa} \ldots \ldots \ldots$. & $0.071 \pm 0.009$ & $0.292 \pm 0.008$ & $0.363 \pm 0.012$ & $0.24 \pm 0.03$ \\
$\mathrm{Bo} \ldots \ldots \ldots$. & $0.048 \pm 0.010$ & $0.149 \pm 0.009$ & $0.197 \pm 0.013$ & $0.32 \pm 0.07$ \\
$\mathrm{AlPa} / 3 \mathrm{Lx} \ldots$ & $0.78 \pm 0.12$ & $0.84 \pm 0.03$ & $0.83 \pm 0.03$ & $\ldots$ \\
$\mathrm{Bo} / 3 \mathrm{Lx} \ldots \ldots$ & $0.53 \pm 0.12$ & $0.43 \pm 0.03$ & $0.45 \pm 0.03$ & $\ldots$ \\
\hline
\end{tabular}

image into a number of statistically independent cells with a typical scale size of the resolution. No statistically significant variations were found.

Because of the morphology of the remnant, we assume that a spherical symmetric hydrodynamical model can only be applied to the northeast part. We choose the Sedov (1959) model for the hydrodynamics of the remnant.

A spatial correlation between the $\mathrm{LE}$ and $\mathrm{ME}$ data is shown in Figure $2 a$. Each point in this figure corresponds to one of the 12 pointing directions (see Fig. 1a) and is obtained as follows. For the observed ME count rate we integrated the spectrum between 2 and $7 \mathrm{keV}$. Higher energies were rejected in order to minimize the possible contribution of galactic ridge emission (see $\S$ III $b$ ). The predicted ME count rate for each pointing was obtained by folding the $3 \mathrm{Lx}$ image with the collimator response of the ME experiment. The errors on both the predicted and observed ME count rates include statistical and systematic errors.

Although a clear correlation between predicted and observed ME count rates is shown, the best fitting line is not acceptable according to $\chi^{2}$ statistics $\left[\chi^{2}=31\right.$ (11 d.o.f.) $]$. This, however, does not necessarily mean that the morphology of RCW 86 is different in different energy bands. In fact, the Einstein IPC image (Pisarski 1984), the IPC energy band being somewhat higher than that of the EXOSAT LE, appears to be very similar to the $3 \mathrm{Lx}$ image.

More likely the difference can be attributed to a radial temperature distribution, as given by the Sedov model. On this basis it is expected that the radial surface brightness profiles in the LE and ME energy band differ. This is a more realistic approach than a constant temperature $T$ for the whole remnant as assumed above. In order to test this hypothesis we corrected the northeast part (which is assumed to be in a Sedov phase) for this difference in surface brightness profiles. We used a NEI plasma model (Jansen 1988) with $\eta=10^{48}, T_{s}=2.6 \mathrm{keV}$ and $N_{\mathrm{H}}=3.3 \times 10^{21} \mathrm{~cm}^{-2}$ (see $\S$ III $b$ ) to calculate the surface brightness profiles. Both the predicted LE and ME surface brightness profiles and the observed surface brightness profile of the northeast part are given in Figure 3. By multiplying the observed $3 \mathrm{Lx}$ image with the, radial dependent, ratio of the theoretical ME and LE surface brightness profiles, one obtains a modified 3Lx image. As shown in Figure $2 b$, this modified $3 \mathrm{Lx}$ image shows an acceptable correlation $\left[\chi^{2}=17(10\right.$ d.o.f. $\left.)\right]$ with the ME data. (The range of $\eta, N_{\mathrm{H}}$ values for which an 


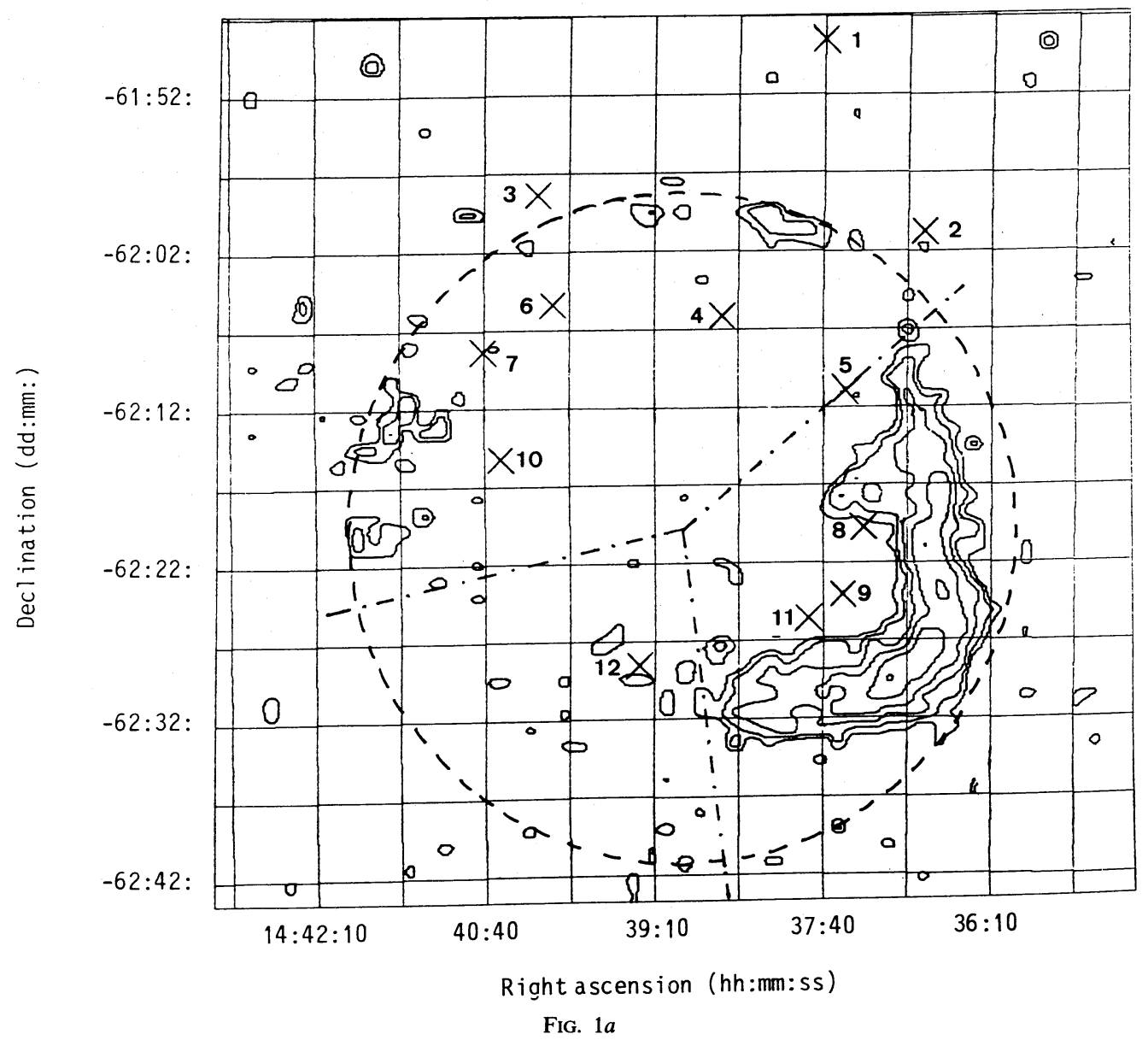

FIG. 1. - (a) The 3Lx image binned into $64^{\prime \prime}$ per pixel. Contour levels: 5.95, 9.40, 14.86, 23.48, 37.09, and $58.61 \times 10^{-4}$ counts s $^{-1}$ pixel $^{-1}$. The crosses represent the pointing directions. The dashed circle defines the best fitted circular shock to the northeast part of the remnant. The dot dashed lines define the angular segments of the northeast and southwest part. (b) The $3 \mathrm{Lx}$ image, binned into $64^{\prime \prime}$ per pixel and smoothed to a resolution of 3 ' (FWHM). Contour levels: 1.49, 4.46, 7.44, 10.42, $13.39,16.37,19.34,22.32,25.30,28.27,31.25$, and $34.22 \times 10^{-4}$ counts s $^{-1}$ pixel $^{-1}$.

acceptable correlation is obtained, is represented by the dotted contour in Figure 5 below).

\section{b) Spectral Information}

The background, necessary for the determination of the $\mathrm{ME}$ spectra, was obtained by either pointing $2^{\circ}$ away from the source or, if these "swop" data were not available, by using slew data, i.e., data obtained during the slew toward and away from the source (see Table 1). Each individual spectrum was corrected for the effect of the ME collimator response. All spectra were corrected for the contribution of galactic ridge emission (Koyama 1984) using the data of Warwick et al. (1985).

In order to find out whether it is justified to add spectra from all pointings, we searched for spectral differences between the northeast and southwest part of the remnant. This was done by calculating hardness ratios using spectra from pointings 1 and 9 (see Fig. 1a). For pointing 1, only $\sim 20 \%$ of the emission is caused by the southwest part whereas for pointing 9 all emission is caused by the southwest part. Although for pointing 1 (northeast part) the hardness ratio is somewhat higher than the hardness ratio of pointing 9 (southwest part), corresponding to a temperature increase of at most $12 \%$, the difference is not significant. This indicates that spectra from all pointings may be added.
Spectral parameters were derived first by fitting the spectrum with a two-component CIE model (Mewe, Gronenschild, and Van den Oord 1985). The interstellar absorption data of Morrison and McCammon (1983) have been used. We fitted the three LE flux points, as determined from the three images, i.e., $3 \mathrm{Lx}, \mathrm{AlPa}$, and $\mathrm{Bo}$, simultaneously with the $\mathrm{ME}$ spectrum, which makes possible a better determination of the spectral parameters and the interstellar hydrogen column density $\left(N_{\mathrm{H}}\right)$. Figure 4 shows the spectrum of RCW 86 together with the best fitting CIE model. The spectral parameters are given in Table 3. The only abundance allowed to vary was that of Fe.

We also used the NEI spectra taken from the figures of Hamilton, Sarazin, and Chevalier (1983) to fit the data. The shape of the Hamilton et al. spectra is a function of $\eta \equiv n_{0}^{2} E_{0}$ ( $n_{0}$ is the density of the unshocked ISM and $E_{0}$ the explosion energy) and the shock temperature $T_{s}$ only. For a number of $\eta$ and $N_{\mathrm{H}}$ values we calculated the NEI count rates for the three images, i.e., $3 \mathrm{Lx}, \mathrm{AlPa}$, and Bo by folding the NEI spectrum with the spectral response of the three filters, respectively. Each spectrum was normalized to the observed ME flux in the 2-10 $\mathrm{keV}$ energy band. For all these fits the shock temperature $T_{s}=$ $T_{e} / 1.3$ has been set to $2.6 \mathrm{keV}$ as obtained from the CIE fit. Since Hamilton et al. use the Sedov model, we fitted only the LE flux of the northeast part of the remnant. The ME flux of the northeast part was determined from the best LE-ME cor- 


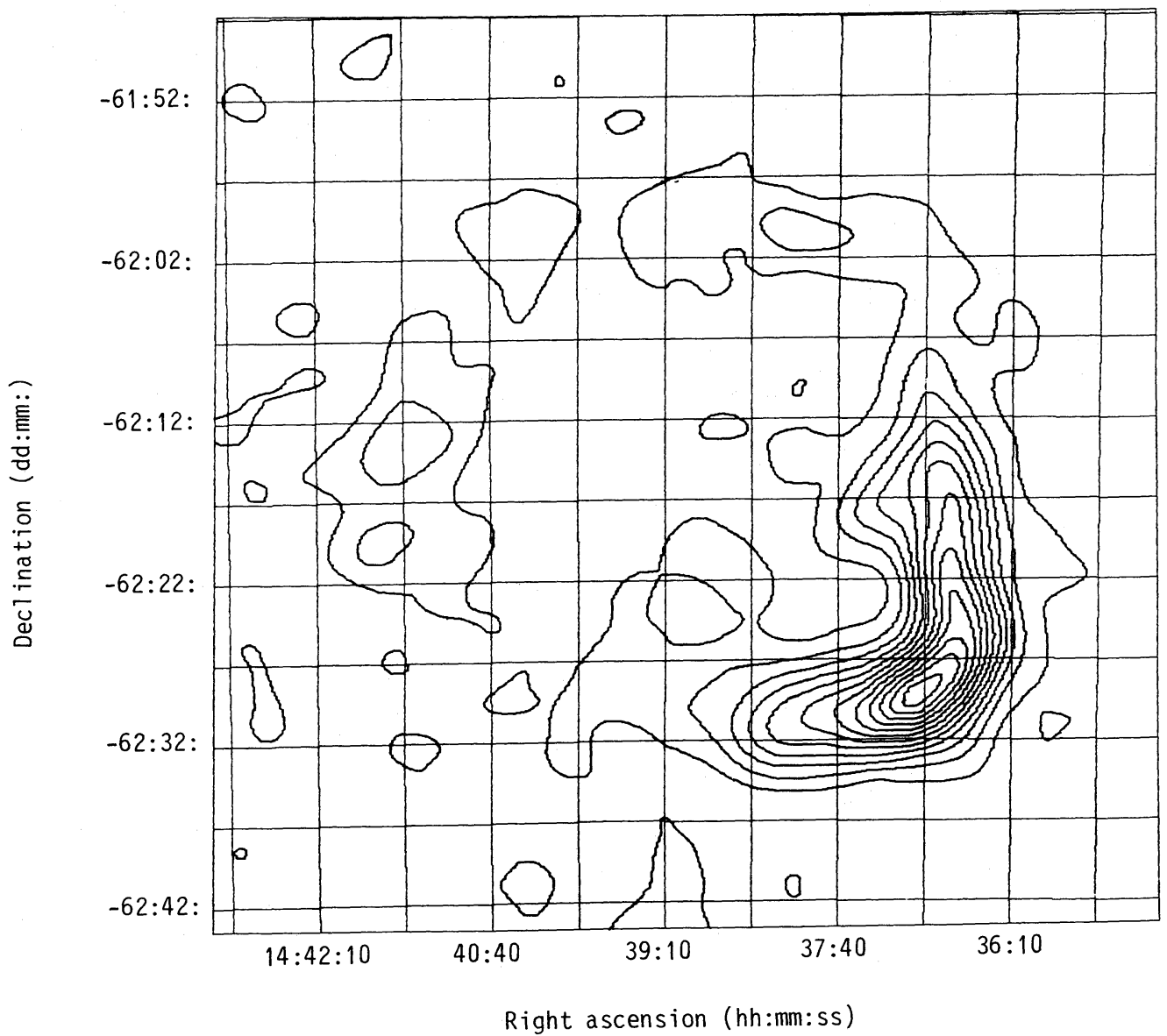

Fig. $1 b$

relation (see $\S$ III $a$ ). The solid contour in Figure 5 shows the acceptable $\eta-N_{\mathrm{H}}$ range obtained this way.

If we compare our spectrum with the $H E A O 1 \mathrm{~A} 2$ spectrum of RCW 86 (Nugent et al. 1984) we find two important differences: (1) The $H E A O$ spectrum is noticeably harder and has a CIE high temperature of $5.1 \mathrm{keV}$ compared with the EXOSAT value of $3.4 \mathrm{keV}$ and (2) the $H E A O$ spectrum has a stronger $\mathrm{FeK} \alpha$ emission line; $\mathrm{EW}_{\mathrm{FeK} \alpha} \approx 1.3 \mathrm{keV}$ compared with EXOSAT: $\mathrm{EW}_{\mathrm{FeK} \alpha} \approx 0.8 \mathrm{keV}$.

These differences can be understood if the background in the $H E A O$ spectrum was not properly subtracted. This seems reasonable since the $H E A O$ background data were obtained in a scanning mode of this experiment. In this case, the galactic ridge flux will not have been subtracted properly, causing an apparent excess flux above $\approx 6 \mathrm{keV}$ and a overestimation of the line strength of the FeK $\alpha$ emission line.

\section{DISCUSSION}

a) Physical Interpretation of the Two CIE Temperature Components

The high-temperature component, as found by fitting with the two-temperature CIE model, is assumed to be associated with the shock in the interstellar medium (e.g., Smith et al. 1988), i.e., the temperature measured equals the electron temperature $T_{e}$ of the swept-up interstellar mass. In that case the shock temperature $T_{s}=T_{e} / 1.3$ (Itoh 1978).

The low-temperature component is possibly caused by NEI effects which, for energies below $2 \mathrm{keV}$, cause enhanced line

TABLE 3

SPECTRAL Fit Parameters ${ }^{a}$

\begin{tabular}{|c|c|}
\hline Parameter & Value \\
\hline $\begin{array}{l}\text { Emission normalization constant } \ldots \ldots \ldots \\
\text { Low-temperature component } \ldots \ldots \ldots \ldots \ldots \\
\text { Emission normalization constant } \ldots \ldots \ldots \ldots \\
\text { High-temperature component } \ldots \ldots \ldots \ldots \ldots \\
\text { Iron abundance } \ldots \ldots \ldots \ldots \ldots \ldots \ldots \ldots \\
\text { Interstellar hydrogen absorption } \ldots \ldots \ldots \ldots \\
\text { Chi-squared } \ldots \ldots \ldots \ldots \ldots \ldots \ldots \ldots \ldots \ldots\end{array}$ & $\begin{aligned} C_{\text {low }} & =(36.9 \pm 1.5) \times 10^{50} \mathrm{~cm}^{-3} \mathrm{pc}^{-2} \\
k T_{\text {low }} & =(0.51 \pm 0.03) \mathrm{keV} \\
C_{\text {high }} & =(10.9 \pm 0.3) \times 10^{50} \mathrm{~cm}^{-3} \mathrm{pc}^{-2} \\
k T_{\text {high }} & =(3.4 \pm 0.2) \mathrm{keV} \\
A_{\mathrm{Fe}} & =0.4 \pm 0.3 \\
N_{\mathrm{H}} & =(1.3 \pm 0.2) \times 10^{21} \mathrm{~cm}^{-2} \\
\chi^{2} & =22.8(19 \text { d.o.f. })\end{aligned}$ \\
\hline
\end{tabular}

a Two-temperature CIE model.

b Relative to solar value. 


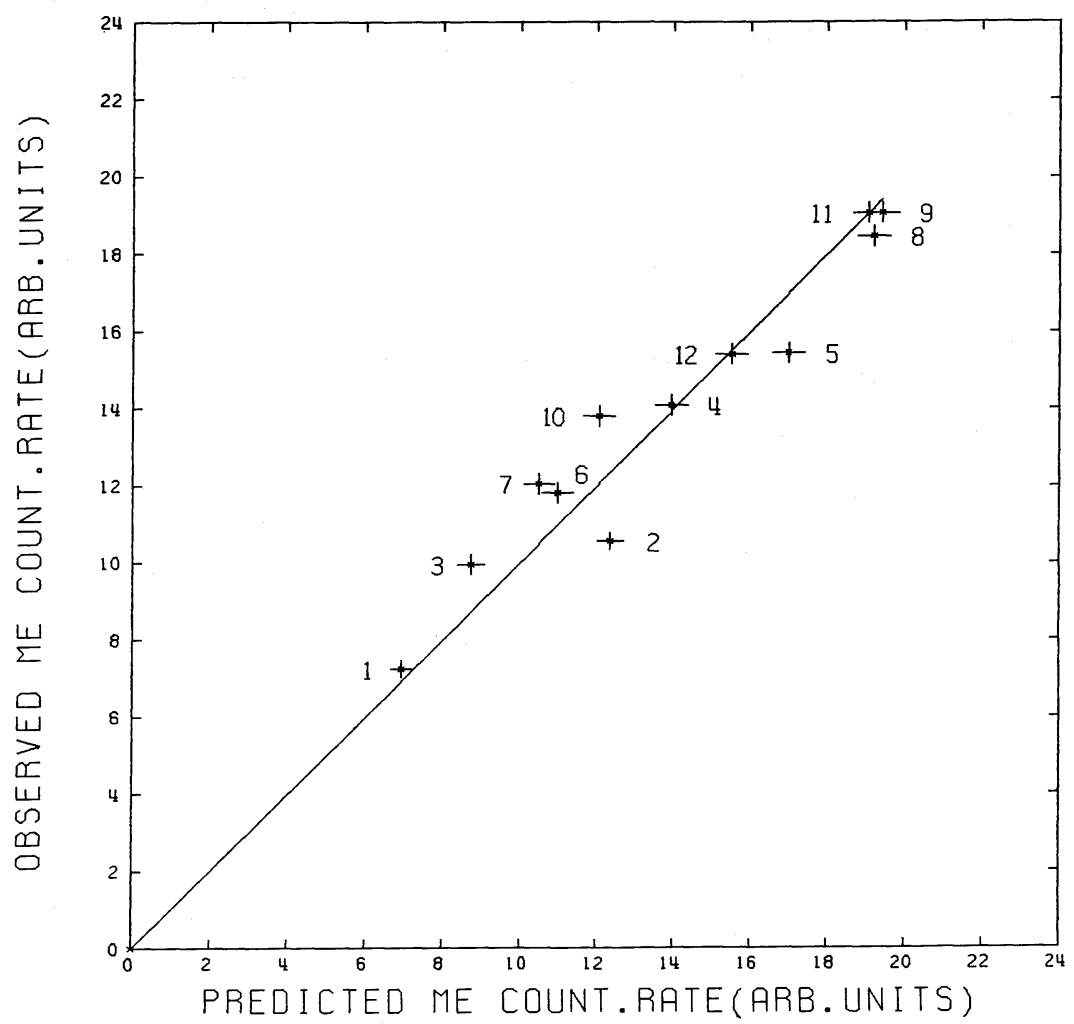

FIG. $2 a$

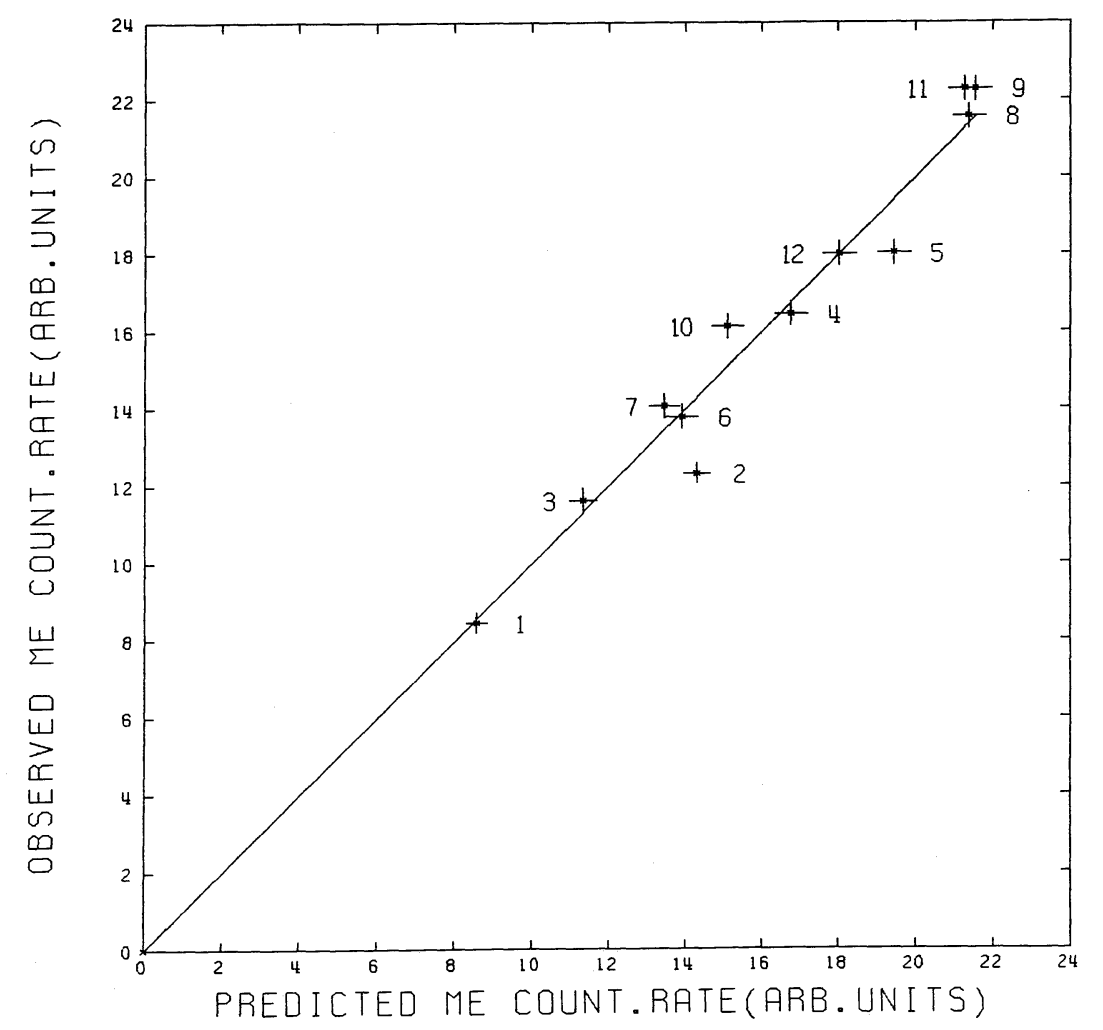

FIG. $2 b$

FIG. 2.-Correlation between observed ME count rates and those predicted on the basis of the observed 3Lx image. (a) The $3 \mathrm{Lx}$ image is used to represent the LE data. (b) In this case the $3 \mathrm{Lx}$ data are modified for the expected differences in the radial surface brightness profiles in different energy bands, as described in the text. 


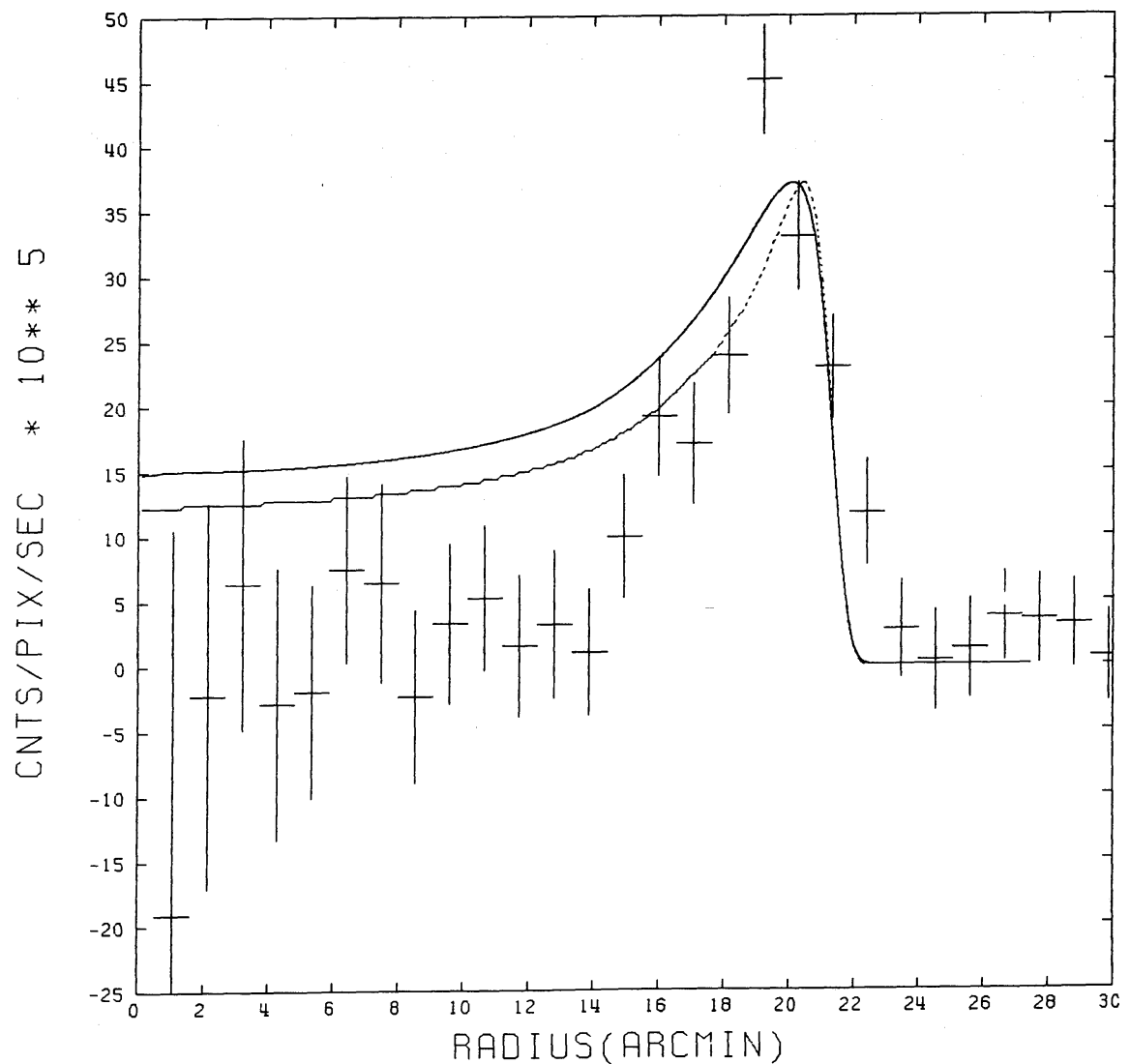

FIG. 3.-The observed radial surface brightness profile (azimuthal average of $3 \mathrm{Lx}$ data) for the northeast part of the remnant together with the theoretical profiles (dashed line, LE; solid line, ME), for $\eta=10^{48} \mathrm{ergs} \mathrm{cm}^{-6}$ and $N_{\mathrm{H}}=3.3 \times 10^{21} \mathrm{~cm}^{-2}$, taking NEI effects into account.

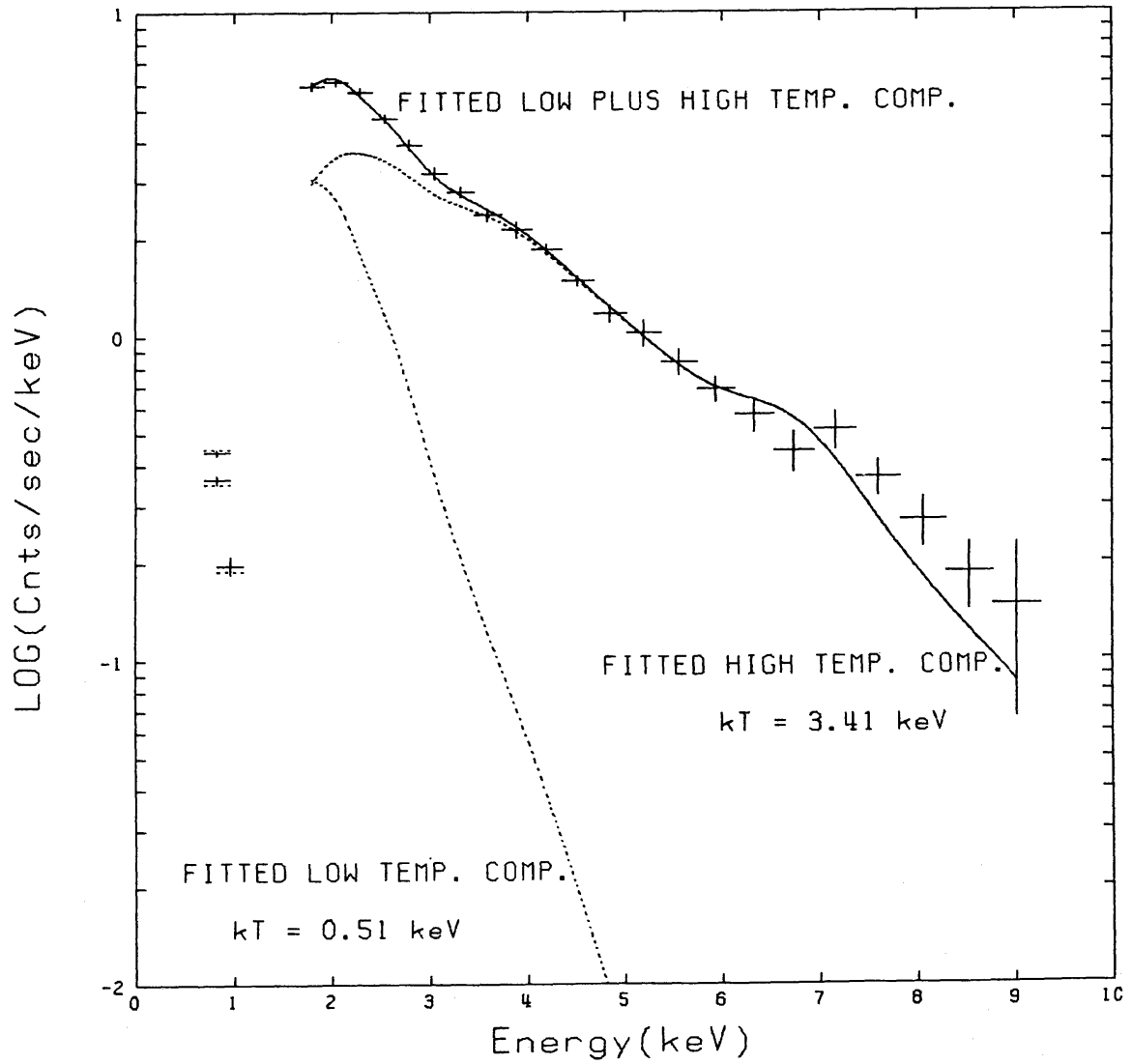

FIG. 4.-LE and ME spectrum of RCW 86. The crosses represent the observed count rates. For ME energies, the upper and lower dotted curves represent the best fitting high- and low-temperature CIE components, respectively. Adding both components gives the continuous curve. For the three LE points the three dotted lines represent the sum of both fitted temperature components. Spectral parameters are given in Table 3.

404 


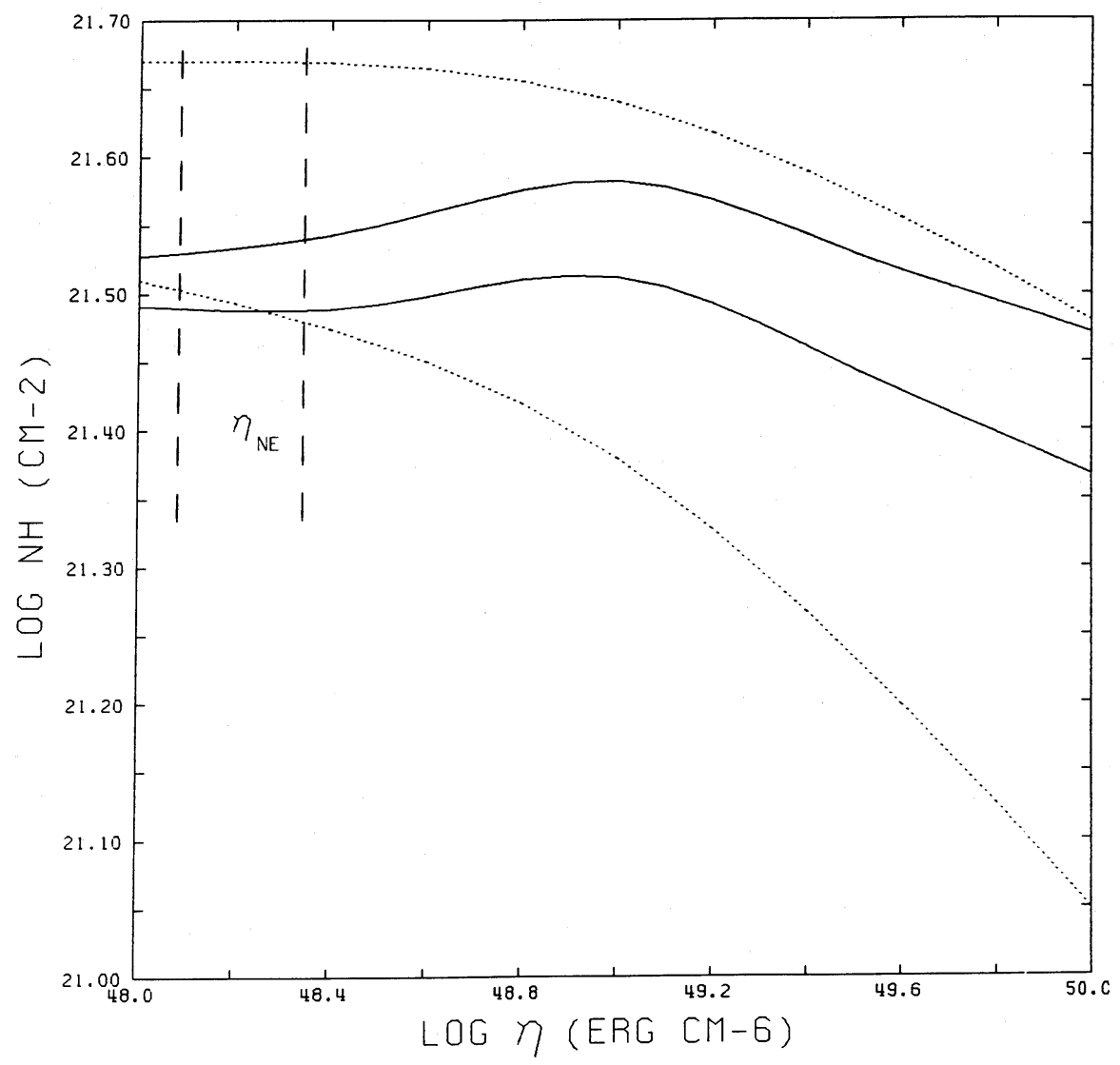

FIG. 5. $-\chi^{2}$-map in the $\log \eta$-log $N_{\mathrm{H}}$ plane. The solid contour represents the $90 \%$ confidence level $\left(\chi_{\min }^{2}+4.61\right)$ obtained from the NEI model fit to the three LE points and the ME flux between 2 and $10 \mathrm{keV}$. The dotted contour represents the $90 \%$ confidence level $\left(\chi_{\min }^{2}+4.61\right)$ obtained from the correlation between the ME data and the modified $3 \mathrm{Lx}$ image. The two vertical dashed lines indicate the range of values of $\eta_{\mathrm{NE}}$ found by applying the Sedov model to the northeast part of the remnant.

emission from elements like $\mathrm{C}, \mathrm{N}, \mathrm{O}$, mimicking a lowtemperature component if the spectrum is observed with insufficient energy resolution. There are three arguments for assuming the existence of NEI. First, the age of the remnant is relatively young, $1800 \mathrm{yr}$, making NEI more likely. A second argument is the acceptable fit to the data with a NEI model. A third argument is given by the acceptable correlation between the low- and medium-energy data when using a NEI model. Therefore we assume that the low-temperature component is not a real physical component but is caused by NEI effects.

\section{b) Hydrodynamics of $R C W 86$}

Before we apply the Sedov model we discuss which scenario led to the observed morphology, especially the difference in intensity between the northeast and southwest part of the remnant as well as the different radius of curvature of the southwest part.

The similar radius of the northeast and the outer extreme of the southwest part together with the similarity between the temperatures of the northeast and southwest part (difference at most $12 \%$ ) are consistent with a Sedov model. The absence of any emission from the central part of the remnant however, is a strong indication for the absence of spherical symmetry as assumed within the Sedov model. The enhanced brightness of the southwest part must be due to a relatively high density since it is not due to a different temperature. Since the maximum extension of the southwest part has about the same radial distance as the northeast part, this relatively high density can have been reached only rather recently. The nonradial shape is most likely caused by the geometry of the denser medium. The southwest part does not show signs of limb brightening. Thus the Sedov model cannot be applied to the southwest part since this model assumes a constant density for the ISM. Another argument against using the Sedov model for the southwest part is given by the clumpy structure seen in the Einstein HRI image (Pisarski et al. 1984), indicating local density variations which may also explain the nonspherical form of the southwest part.

When we apply the Sedov model to the northeast part of the remnant we use the following parameters: (1) the shock temperature $T_{s}=T_{e} / 1.3=(2.6 \pm 0.2) \mathrm{keV}$; (2) the shock radius $\theta=\left(21^{\prime} \pm 1^{\prime}\right)$ (see Fig. 3$)$; (3) the emission normalization constant $\left(C_{\mathrm{high}}\right)_{\mathrm{NE}}$ is a fraction of the total emission normalization constant $C_{\text {high }}$. This fraction, determined from the LE-ME correlation, equals $0.30 \pm 0.04$ which gives $\left(C_{\mathrm{high}}\right)_{\mathrm{NE}}=(3.3 \pm 0.4)$ $\times 10^{50} \mathrm{~cm}^{-3} \mathrm{pc}^{-2}$; (4) for the age of $\mathrm{RCW} 86$ we use the generally accepted age as found from old Chinese records: $t=1800 \mathrm{yr}$. Using these parameters together with the basic 
expressions as given in the Appendix, we derive the following parameters:

$$
\begin{aligned}
\left(r_{s}\right)_{\mathrm{NE}} & =(6.7 \pm 0.2) \mathrm{pc} \\
d & =(1.1 \pm 0.1) \mathrm{kpc}, \\
\left(n_{0}\right)_{\mathrm{NE}} & =(0.11 \pm 0.01) \mathrm{cm}^{-3}, \\
E_{0} & =(1.5 \pm 0.2) \times 10^{50} \mathrm{ergs}, \\
M_{s} & =(4.9 \pm 0.5) M_{\odot}, \\
(\eta)_{\mathrm{NE}} & =(1.7 \pm 0.5) \times 10^{48} \mathrm{ergs} \mathrm{cm}^{-6},
\end{aligned}
$$

in which $\left(r_{s}\right)_{\mathrm{NE}}$ is the shock radius for the northeast part, $d$ the distance to the remnant, $\left(n_{0}\right)_{\mathrm{NE}}$ the density of the unshocked ISM in the northeast region, $E_{0}$ the explosion energy, $M_{s}$ the total swept-up interstellar mass and $(\eta)_{\mathrm{NE}}$ the characteristic NEI parameter for the northeast part. In deriving these parameters we assumed a filling factor 0.4 for the northeast part which is obtained from the position angle which defines the northeast part. The value of $(\eta)_{\mathrm{NE}}$ falls inside the range of $\eta$ 's determined by the contours in Figure 5 . In that case $N_{\mathrm{H}}$ equals $(3.3 \pm 0.2) \times 10^{21} \mathrm{~cm}^{-2}$.

Parameters for the southwest part can be obtained by applying $E_{0}$, as found for the northeast part, also to the southwest. By using $(V)_{\mathrm{sw}}=(6.4 \pm 3.7) \times 10^{55} \mathrm{~cm}^{3}$ for the emitting volume of the southwest part, a value obtained from the Einstein HRI image because of its relative high angular resolution, and $C_{\text {high }}=(7.6 \pm 0.5) \times 10^{50} \mathrm{~cm}^{-3} \mathrm{pc}^{-2}$, we now find for the southwest part

$$
\begin{aligned}
\left(n_{0}\right)_{\mathrm{sw}} & =(1.1 \pm 0.3) \mathrm{cm}^{-3}, \\
(\eta)_{\mathrm{sw}} & =(1.7 \pm 1.0) \times 10^{50} \mathrm{ergs} \mathrm{cm}^{-6} .
\end{aligned}
$$

The expected equivalent width of the $\mathrm{FeK} \alpha$ emission line of $0.40 \mathrm{keV}$ corresponding to this $(\eta)_{\mathrm{sw}}$ (see Fig. 15 in Hamilton $e$ al. 1983), is consistent with the observed value of $(0.8 \pm 0.6)$ $\mathrm{keV}$. This indicates that $\mathrm{EW}_{\mathrm{FeK} \alpha}$ is mainly determined by the emission from the southwest part.

The obtained value of $\left(n_{0}\right)_{\text {sw }}$ is not in disagreement with the value obtained from the analysis of the infrared IRAS data of the southwest part of the remnant, i.e., $n_{0} \approx 0.7 \pm 0.2 \mathrm{~cm}^{-3}$ (Greidanus 1989). This value is obtained by using the densitydependent relation between dust- and electron temperature of the shocked gas (Dwek 1987).

The distance found above falls well inside the wide range of distances given by several authors, the wide range being caused by the different data and methods used.

We check whether the use of a Sedov phase for the northeast part is justified by using the characteristic parameters of Types I and II SN explosions:

$$
\begin{gathered}
\text { Type I: } E_{0} \approx 10^{51} \mathrm{ergs}, M_{\mathrm{ej}} \approx 1 M_{\odot} ; \\
\text { Type II: } E_{0} \approx 10^{51} \mathrm{ergs}, M_{\mathrm{ej}} \approx 5-15 M_{\odot} ;
\end{gathered}
$$

in which $M_{\text {ej }}$ is the ejected stellar mass (Woosley and Weaver 1986; Pisarski et al. 1984). In case of a Sedov phase, the assumption $M_{\text {sw }}>M_{\text {ej }}$ must hold. We conclude that this is the case only if RCW 86 is the remainder of a Type I explosion. The explosion energy found from our Sedov analysis is lower than the canonical value of $10^{51}$ ergs. About the same low value for $E_{0}$ is also found from the Tycho analysis (Smith et al. 1988), indicating that the explosion energy for a Type I remnant might be a few times $10^{50}$ ergs. The possibility, however, that RCW 86 is a Type II remnant cannot be excluded but in that case it must be in a free expansion phase (Pisarski et al. 1984).

\section{c) Emission from a Reverse Shock}

When we fitted a NEI model to our data (see $\S$ III $b$ ) we neglected the eventual flux contribution from a reverse shock. This flux contribution can be calculated for a Type I remnant by using the hydrodynamical model of Chevalier (1982) which assumes that the expanding ejecta have a power-law density profile $\rho \propto r^{-7}$ (based on the theoretical modeling of Type I supernovae). This model predicts a ratio between the temperatures and emission normalization constants for the primary blastwave and reverse shock $\sim 2.4$ and 1.2 , respectively. We therefore find

$$
\begin{aligned}
\left(C_{\mathrm{rev}}\right)_{\mathrm{NE}} & \approx 2.8 \times 10^{50} \mathrm{~cm}^{-3} \mathrm{pc}^{-2}, \\
\left(k T_{\mathrm{rev}}\right)_{\mathrm{NE}} & \approx 1.4 \mathrm{keV},
\end{aligned}
$$

where $\left(C_{\text {rev }}\right)_{\mathrm{NE}}$ and $\left(k T_{\mathrm{rev}}\right)_{\mathrm{NE}}$ are the emission normalization constant and electron temperature of the reverse shock, respectively. Taking $\eta=1.7 \times 10^{48}$ ergs $\mathrm{cm}^{-6}$ for the northeast part and subsequently $N_{H}=3.3 \times 10^{21} \mathrm{~cm}^{-2}$ (see Fig. 5), we find a flux contribution from the reverse shock in the LE energy range of $\sim 7 \%$ of the observed LE flux. This indicates that neglecting the reverse shock flux hardly influences the results obtained earlier. Using Chevalier's model we find for the ejected mass $M_{\text {ej }} \approx 2.5 M_{\odot}$ which is quite high for a Type I remnant. However, the amount of ejected mass is a strong function of the power-law density profile of the ejecta. In case $\rho \propto r^{-6}$ we find that the reverse shock produces less than $7 \%$ of the observed LE flux whereas $M_{\mathrm{ej}} \approx 1.4 M_{\odot}$ which is much more like the typical mass for a Type I remnant.

\section{v. CONCLUSIONS}

X-ray observations of RCW 86 reveal the following properties:

1. The remnant, for which a distance of $\sim 1.1 \mathrm{kpc}$ is derived, consists of two parts; in the northeast part the shockwave is running through a constant density ISM; the southwest part of the shockwave has recently moved into a region of relatively high density. There is no direct indication for any large temperature difference between the shocked gas in the northeast and southwest part of the remnant. Both regions have an electron temperature of $\sim 3.4 \mathrm{keV}$.

2. The low energy flux in the northeast part is most likely caused by NEI effects in the shocked plasma. Using the NEI model of Hamilton et al. (1983), the northeast part can be described by a NEI model with $\eta=1.7 \times 10^{48} \mathrm{ergs} \mathrm{cm}^{-6}$.

3. For the southwest part, the density of the unshocked ISM is much higher, $n_{0} \approx 1.1 \mathrm{~cm}^{-3}$, than for the northeast part, $n_{0} \approx 0.11 \mathrm{~cm}^{-3}$.

4. Since we do not find any temperature difference between the northeast and southwest part, the radius of curvature change of the southwest part compared to the northeast part cannot be due to a reduction of the shock velocity $v_{s}\left(T_{s} \sim v_{s}^{2}\right)$. Therefore this change of curvature must just reflect the shape of the clouds that are brightened up.

5. Our results are consistent with the assumption of $\mathrm{RCW}$ 86 being the remainder of a Type I SN for which the northeast part is in the Sedov phase of evolution.

The Laboratory for Space Research Leiden is financially supported by the Netherlands Organization for the Advancement of Scientific Research. 


\section{APPENDIX}

\section{THE HYDRODYNAMICAL SEDOV MODEL}

The Sedov model describes the uniform expansion of a shock wave, caused by a pointlike explosion into a homogeneous interstellar medium (Sedov 1959). Here we give some useful relations, using the notation of Hamilton et al. (1983):

$$
\begin{aligned}
v_{s} & =839 T_{7}^{1 / 2} \mathrm{~km} \mathrm{~s}^{-1}, \\
t & =4015 \eta_{51}^{-1 / 6} T_{7}^{-5 / 6} E_{51}^{1 / 2} \mathrm{yr}, \\
r_{s} & =8.62 \eta_{51}^{-1 / 6} T_{7}^{-1 / 3} E_{51}^{1 / 2} \mathrm{pc} \\
M_{s} & =96.8 T_{7}^{-1} E_{51} M_{\odot} \\
\eta_{51} & =n_{0}^{2} E_{51} \operatorname{ergs~} \mathrm{cm}^{-6}
\end{aligned}
$$

$v_{s}$ is the velocity of the shockfront, $n_{0}$ is the density of the unshocked ISM, $t$ is the age of the remnant, $r_{s}$ is the radius of the shockfront, $M_{s}$ is the amount of swept up interstellar mass, $T_{7}$ is the shock temperature in units of $10^{7} \mathrm{~K}$, and $E_{51}$ is the explosion energy in units of $10^{51}$ ergs. Combining relations (A2), (A3), and (A5) gives

$$
\begin{aligned}
\frac{r}{t} & =2.147 T_{7}^{1 / 2} \times 10^{-3} \mathrm{pc} \mathrm{yr}^{-1} \\
E_{51} & =\left(\frac{t}{4015}\right)^{3} n_{0} T_{7}^{5 / 2} \mathrm{ergs}
\end{aligned}
$$

The ISM density $n_{0}$ can be found by combining the emission normalization constant $C$, given by the spectral fit and the distance $d$

$$
C d_{\mathrm{pc}}^{2}=\int n_{e} n_{\mathrm{H}} d V
$$

in which for a Sedov remnant

$$
\int n_{e} n_{\mathrm{H}} d V=2.07\left(\frac{n_{e}}{n_{\mathrm{H}}}\right) n_{0}^{2} \frac{4}{3} \pi r_{\mathrm{cm}}^{3} f
$$

The filling factor $f$ equals 1 in case of a totally filled shell-like remnant.

\section{REFERENCES}

Caswell, J. L., Clark, D. H., and Crawford, D. F. 1975, Australian J. Phys. Suppl., 37, 39.

Chevalier, R. A. 1982, Ap. J., 258, 790 .

Clark, D. H., and Caswell, J. L. 1976, M.N.R.A.S., 174, 267.

Clark, D. H., and Stephenson, F. R. 1977, Historical Supernovae (Oxford: Pergamon).

de Korte, P. A. J., et al. 1981, Space Sci. Rev., 30, 495.

Dwek, E. 1987, Ap. J., 322, 812.

Greidanus, H. 1989, in preparation.

Hamilton, A. J. S., Sarazin, C. L., and Chevalier, R. A. 1983, Ap. J. Suppl., 51, 115.

Hill, E. R. 1967, Australian J. Phys., 20, 297.

Ilovaisky, S. A., and Lequeux, J. 1972, Astr. Ap., 18, 169.

Itoh, H. 1977, Pub. Astr. Soc. Japan, 29, 813. 1978, Pub. Astr. Soc. Japan, 30, 489. 1979, Pub. Astr. Soc. Japan, 31, 541

Jansen, F. A. 1988, Ph.D. thesis, University of Leiden.

Koyama, K. 1984, in X-Ray Astronomy, ed. M. Oda and R. Giacconi (Tokyo:

Institute of Space and Astronautical Science), p. 325.

Mewe, R., Gronenschild, E. H. B. M., and Van den Oord, G. H. J. 1985, Astr. Ap. Suppl., 62, 197.

Milne, D. K. 1970, Australian J. Phys., 23, 425.
Minkowski, R. 1968, in Stars and Stellar Systems, Vol. 7, ed. B. M. Middlehurst and L. H. Aller (Chicago: University of Chicago Press), p. 623.

Morrison, R., and McCammon, D. 1983, Ap. J., 270, 119.

Naranan, S., Shulman, S., Yentis, D., Fritz, G., and Friedman, H. 1977, Ap. J. (Letters), 213, L53.

Nugent, J. J., Pravdo, S. H., Garmire, G. P., Becker, R. H., Tuohy, I. R., and Winkler, P. F. 1984, Ap. J., 284, 612 .

Pisarski, R. L., Helfand, D. J., and Kahn, S. M. 1984, Ap. J., 277, 710.

Poveda, A., and Woltjer, L. 1968, Astr. J., 73, 65.

Ruiz, M. T. 1981, Ap. J., 243, 814.

Sedov, L. I. 1959, Similarity and Dimensional Methods in Mechanics (New York: Academic).

Smith, A., Davelaar, J., Peacock, A., Taylor, B. G., Marini, M., and Robba, N. R. 1988, Ap. J., 325, 288.

Turner, M. J. L., Smith, A., and Zimmerman, H. U. 1981, Space Sci. Rev., 30, 513.

Warwick, R. S., Turner, M. J. L., Watson, M. G., and Willingale, R. 1985, Nature, 317, 218.

Westerlund, B. F. 1969, Ap. J., 74, 879.

Winkler, P. F. 1978, Ap. J., 221, 220.

Woosley, S. E., and Weaver, T. A. 1986, Ann. Rev. Astr. Ap., 24, 205.

J. J. ClaAs and J. S. KAASTRA: Laboratory for Space Research Leiden, Niels Bohrweg 2, 2300 RA Leiden, The Netherlands

P. A. J. DE KORTE: Laboratory for Space Research Utrecht, Beneluxlaan 21, 3527 HS Utrecht, The Netherlands

A. Smith and A. Peacock: Space Science Department of ESA, ESTEC, Postbus 299, 2200 AG Noordwijk, The Netherlands 